\title{
¿Puede el fentanilo intratecal disminuir la aparición de náuseas y vómito en comparación con ondansetrón intravenoso durante cesárea electiva? Revisión sistemática de la literatura y metaanálisis
}

\author{
Can intrathecal fentanyl decrease the occurrence of nausea \\ and vomiting compared with intravenous ondansetron \\ during elective cesarean section? Literature Systematic \\ review and meta-analysis
}

Andrés Fabricio Caballero-Lozada MD1,2, Alejandro Botero-Fernández MD², Charlie Castillo-Rivera MD², Andrés Zorrilla-Vaca B.Sc ${ }^{2}$

\begin{abstract}
Elective caesarean section is one of the surgeries with the highest intraoperative incidence of nausea, retching and vomiting (IONV), due, among other causes, to the use of anesthetics during the procedure. Some clinical trials have associated the use of low-dose intrathecal (IT) fentanyl with a lower incidence of nausea, retching and vomiting compared to other anesthetics used during caesarean sections. In this context, the objective of this meta-analysis was to evaluate the decrease in the appearance of nausea and vomiting during elective caesarean section with the application of IT fentanyl when compared with the use of intravenous ondansetron (EV). A systematic search was conducted in the main databases (PubMed, EMBASE, ClinicalTrials.gov, Cochrane Library
\end{abstract}

Key words:

Fentanyl, ondansetron, nausea, caesarean section

Departamento de Anestesiología, Universidad del Valle. Cali, Colombia. Hospital Universitario del Valle. Cali, Colombia. Autor de correspondencia: Andrés Fabricio Caballero-Lozada, Departamento de Anestesiología y Reanimación. Universidad del Valle. Cali, Colombia.

Fecha de recepción: 20 de marzo de 2020

Fecha de aceptación: 14 de agosto de 2020

ORCID

http://orcid.org/0000-0002-4161-4805

Financiamiento:

No se recibieron aportes económicos para la realización del presente estudio.

Conflicto de intereses:

Los autores declaran no tener conflicto de intereses.

Correspondencia

Andrés Fabricio Caballero-Lozada

andres.caballero@correounivalle.edu.co. 
and Google Scholar) for Randomized Clinical Trials (RCTs) that evaluated the use of IT fentanyl compared to ondansetron EV to decrease the occurrence and incidence of IONV during elective caesarean section. The meta-analysis showed a reduction in the incidence of nausea (RR 0.52, 95\% Cl 0.29-0.93, P $=0.03)$, gagging (RR $0.39,95 \% \mathrm{Cl} 0,18-0.88, P=0.02)$ and vomiting (RR 0.26 , $95 \% \mathrm{Cl} 0.11-0.64, \mathrm{P}=0.003)$ in the group of patients treated with IT fentanyl compared to the group treated with EV ondansetron. From the results, it is suggested that the administration of 12.5 to $20 \mu \mathrm{g}$ of IT fentanyl may decrease the incidence of IONV in patients undergoing elective caesarean section, although the importance of more high-quality RCTs is highlighted.

\section{RESUMEN}

La cesárea electiva es una de las cirugías con mayor incidencia intraoperatoria de náuseas, arcadas y vómito (NAV), debido entre otras causas, al uso de anestésicos durante el procedimiento. Algunos ensayos clínicos han asociado el uso de fentanilo intratecal (IT) a dosis bajas con una menor incidencia de náuseas, arcadas y vómito en comparación con otros anestésicos usados durante las cesáreas. En este contexto el objetivo de este metaanálisis fue evaluar la disminución en la aparición de náuseas y vómito durante cesárea electiva con la aplicación de fentanilo IT al compararlo con el uso de ondansetrón intravenoso (EV). Se realizó una búsqueda sistemática en las principales bases de datos (PubMed, EMBASE, ClinicalTrials.gov, Cochrane Library y Google Scholar) para ensayos clínicos aleatorizados (ECA) que evaluaron el uso del fentanilo IT en comparación con ondansetrón EV para disminuir la aparición e incidencia de IONV durante cesárea electiva. En el metaanálisis se evidenció una reducción en la incidencia de náusea (RR 0,52,95\% IC 0,29-0,93, $P=0,03$ ), arcada (RR $0,39,95 \%$ IC $0,18-0,88, P=0,02$ ) y vómito (RR 0,26, 95\% IC 0,11-0,64, $P=$ 0,003 ) en el grupo de pacientes tratados con fentanilo IT comparado con el grupo tratado con ondansetrón EV. A partir de los resultados, se sugiere que la administración de 12,5 a $20 \mu \mathrm{g}$ de fentanilo IT puede disminuir la incidencia de NAV intraoperatorias en pacientes sometidas a cesárea electiva, aunque se resalta la importancia de más ECA de alta calidad.

\section{Palabras clave:}

Fentanilo intratecal, ondansetrón, náusea, cesárea

\section{Intorducción}

as náuseas, arcadas y vómitos (NAV) intraoperatorios que afectan a las mujeres que se someten a anestesia regional para una cesárea son un problema clínico importante ya que estas técnicas se utilizan ampliamente[1]. Estos síntomas, además de causar incomodidad a la mujer que está dando a luz, perjudica las condiciones quirúrgicas para el ginecólogo y puede llegar a inducir efectos secundarios como la aspiración de contenido gástrico, aumento de dolor intra y postoperatorio, e incluso sangrado o trauma quirúrgico[1]. Las náuseas y los vómitos en el postoperatorio (NVPO) de la anestesia epidural son un tema ampliamente estudiado, sin embargo, las
NAV intraoperatorias no se han estudiado bien[2]. Las náuseas y los vómitos son problemas comunes en una variedad de operaciones quirúrgicas, sin embargo, es más frecuente en las cesáreas bajo anestesia regional, de hecho, según estudios observacionales es una complicación que se presenta con una incidencia entre $20 \%$ y $30 \%$ de la población general, llegando hasta un $80 \%$ en pacientes de alto riesgo[3]. Entre los factores que contribuyen al NAV intraoperatorias se encuentran el aumento de la presión intragástrica, la hipotensión, la tracción del peritoneo, la manipulación quirúrgica excesiva y la estimulación visceral, el uso de opioides, los agentes uterotónicos y el estado mental del paciente[2],[3]. De acuerdo a Semiz et al.[2], el hecho de que las NAV intraopera- 
torias se observen con menos frecuencia con la edad materna avanzada se atribuye a la disminución de los niveles de estrógenos.

Por lo anterior, se han planteado diversas estrategias anestésicas encaminadas a obtener menores tasas de NV intra y postoperatoria para lograr una rápida recuperación. En algunos países, como el Reino Unido, existe una recomendación para la administración rutinaria de medicamentos antieméticos o acupuntura para tratar de prevenir las náuseas y los vómitos en la cesárea[4]. Sin embargo, esta práctica clínica es más aplicada como tratamiento, y no como profilaxis. Estas intervenciones ya sea de tratamiento o profilaxis, deben siempre aplicarse basadas en evidencia, puesto que están asociadas con mayores costos de atención médica y riesgos potenciales para la mujer y el recién nacido[4]. Algunos ensayos clínicos han asociado el uso de fentanilo IT a dosis bajas con una menor incidencia de NAV intraoperatorias en comparación con placebo, con resultados alentadores para el fentanilo IT. Por otro lado, otros estudios han encontrado que el fármaco antiemético ondansetrón es eficaz durante el período intraoperatorio y postoperatorio para la cesárea bajo anestesia espinal[5]. En este contexto, el objetivo de este metaanálisis fue evaluar la disminución en la aparición de NAV intraoperatorias durante cesárea electiva con la aplicación de fentanilo IT al compararlo con el uso de ondansetrón EV.

\section{Metodología}

El presente metaanálisis fue realizado bajo los parámetros PRISMA, así como con las recomendaciones de la Colaboración Cochrane. Se realizó una búsqueda en bases de datos electrónicas sin restricciones en idioma, y los estudios extranjeros fueron traducidos. Se adaptó la estrategia de búsqueda para las bases de datos Pubmed (1975 a 4 de noviembre de 2018), Embase (1975 a 4 de noviembre de 2018), ClinicalTrials.gov (1975 a 4 de noviembre de 2018), Cochrane Library (1975 a 4 de noviembre de 2018) y Google Scholar (1975 a 4 de noviembre de 2018). Los términos utilizados en la búsqueda en las bases de datos al igual que la ecuación se encuentran en la Tabla 1.

Los artículos obtenidos en la búsqueda de litera- tura fueron revisados de forma independiente por 2 de los investigadores, quienes realizaron la selección de estudios según los criterios de elegibilidad, inicialmente por título y resumen y, posteriormente, con el texto completo. El diagrama en la Figura 1 resume la selección de los estudios.

\section{Criterios de elegibilidad}

Se seleccionaron ensayos clínicos aleatorizados (ECA) evaluando la incidencia de NAV intraoperatorias con el uso de fentanilo IT en comparación al uso de ondansetrón EV. No se impusieron restricciones de idioma o fecha de publicación. El tipo de participantes eran: Mujeres ASA I y II, en estado de embarazo, programadas para cesárea electiva por cualquier causa. Los estudios debían comparar la aparición de NAV intraoperatorias en pacientes que recibieron fentanilo IT u ondansetrón EV. Como tipo de medición de desenlaces, se consideraron como desenlaces primarios la aparición de NAV intraoperatorias y se excluyeron estudios que no reportaron la frecuencia absoluta o relativa de NVA.

\section{Proceso de recolección de datos}

Dos investigadores (A.B. y A.F.C.) examinaron de forma independiente cada estudio extrayendo información esencial (tipo de estudio, primer autor, tamaño de muestra, incidencia de NAV intraoperatorias, dosis de fentanilo IT, dosis de bupivacaína usada para anestesia espinal, dosis de ondansetrón EV) en una base de datos previamente diseñada en Excel. En cada estudio se obtuvieron para análisis los datos correspondientes a frecuencias (absoluta y relativa) de náuseas, arcadas y vómito. El riesgo de sesgo en los estudios se determinó mediante la herramienta para evaluación de riesgo Cochrane.

El objetivo primario fue evaluar la disminución de NAV intraoperatorias durante la cesárea electiva con la administración de fentanilo IT, por lo cual se calculó el riesgo relativo para cada evento con un intervalo de confianza del 95\%. Se evaluó la heterogeneidad en los estudios mediante test de $\chi^{2}$ y el riesgo de sesgo entre los estudios se presenta en una gráfica de embudo y se evaluó también la heterogeneidad de

\section{Tabla 1. Criterios de búsqueda en bases de datos}

1. Fentanyl (Mesh) AND ondansetron (Mesh) AND nausea and vomiting, intraoperative (Mesh) AND spinal anesthesia (Mesh)

2. Fentanyl AND ondansetron AND nausea, intraoperative

3. "Spinal fentanyl" AND "intravenous ondansetron" AND nausea AND vomiting 


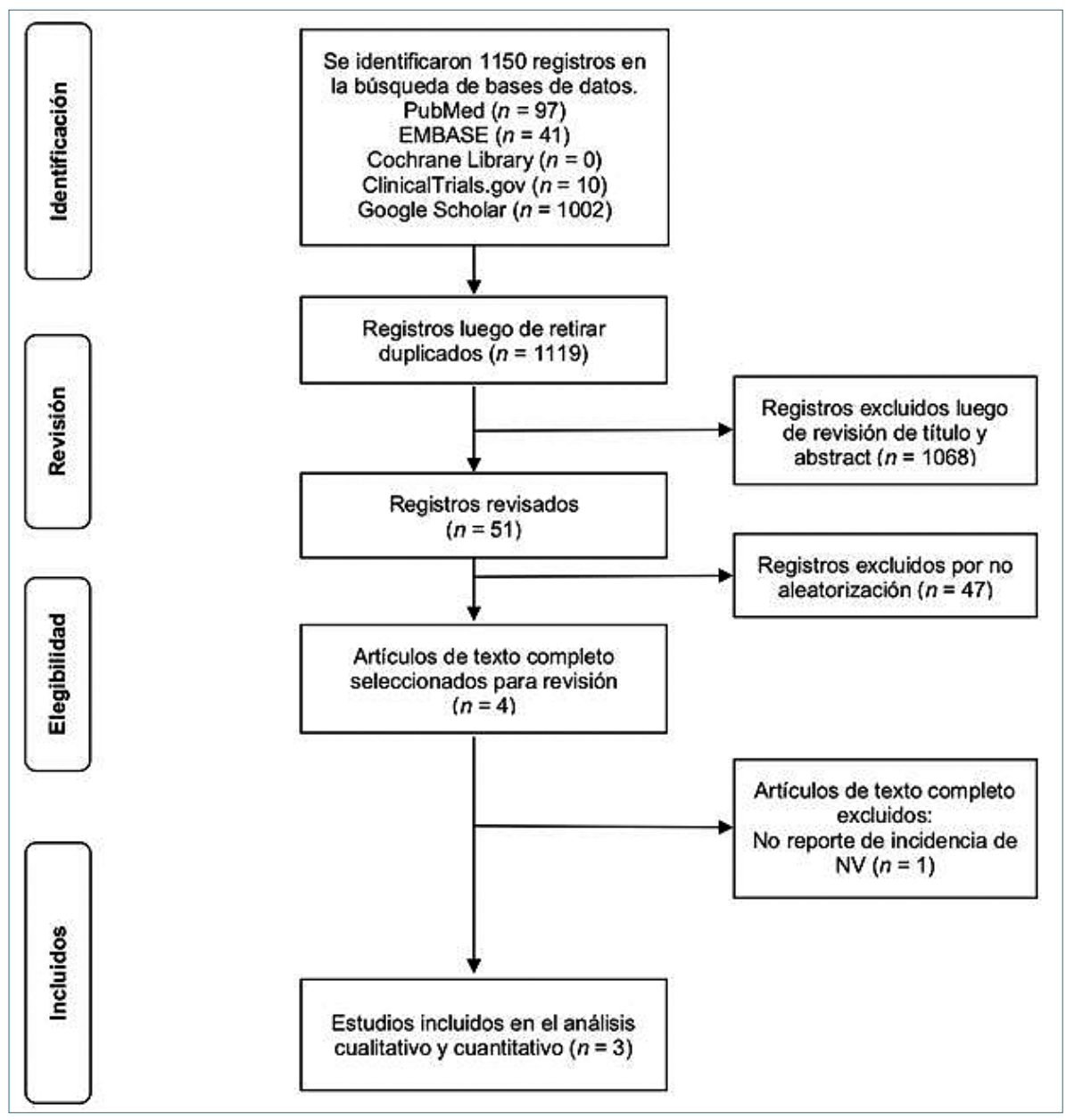

Figura 1. Diagrama de flujo PRISMA de la selección de los estudios.

los mismos en el análisis cuantitativo. No se realizaron análisis adicionales al objetivo primario.

\section{Resultados}

Un total de tres ECA fueron incluidos en el análisis cuantitativo, obteniendo un total de 320 pacientes. Todos los estudios compararon dos grupos de pacientes según el tratamiento recibido: fentanilo IT y ondansetrón EV, administrados durante la aplicación de anestesia espinal. Dos de los estudios utilizaron 12,5 $\mu \mathrm{g}$ de fentanilo IT, mientras que en el tercer estudio los autores utilizaron una dosis de $20 \mu \mathrm{g}$ de fentanilo IT. En la Tabla 2 se encuentran resumidas las principales características de los artículos incluidos.

La evaluación del riesgo de sesgo mostró que los tres estudios incluidos eran de calidad razonable, siendo la deficiencia más común la falta de cegamiento del paciente y del personal del estudio, seguido del cegamiento de los resultados de la evaluación (Figura $3)$, esto ocurre a menudo en este tipo de estudios, siendo una limitación difícil de evitar en procedimientos de precisión.

\section{Metaanálisis}

En total se obtuvo información de 320 pacientes, con rango de edad de 20-35 años (Tabla 3), con una clasificación del estado físico ASA I y II, programadas para cesárea electiva y que fueron sometidas a anestesia espinal con bupivacaína pesada entre 10[6,7] y 12 mg[8]: En total, 160 pacientes recibieron ondansetrón EV y 160 recibieron fentanilo IT, y para cada grupo se evaluó la aparición de NAV intraoperatorias (Tabla 3).

En el metaanálisis de los tres estudios se evidenció 


\begin{tabular}{lccc} 
& Tabla 2. Características de los estudios incluidos en el metaanálisis & \\
\hline Estudio & $\mathbf{n}$ & Intervención & Control \\
Gowda (2014)[6] & 120 & Fentanilo IT & Ondansetrón EV \\
Jain (2015)[7] & 60 & Fentanilo IT & Ondansetrón EV \\
Soreingam (2017)[8] & 140 & Fentanilo IT & Ondansetrón EV \\
\hline
\end{tabular}

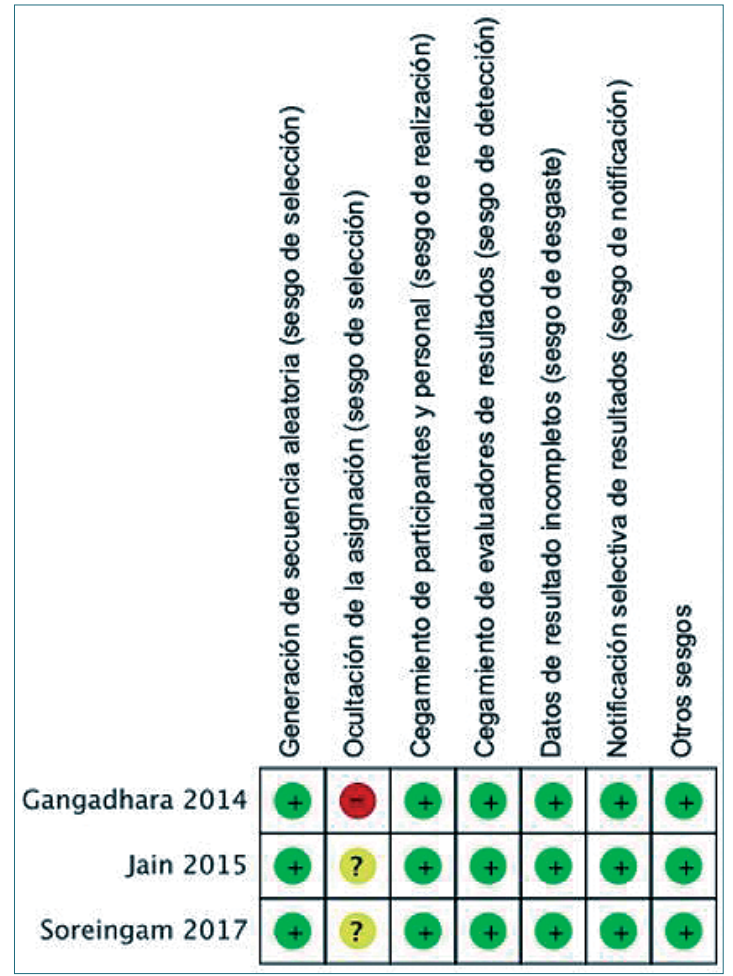

Figura 2. Evaluación del riesgo de sesgo. una reducción estadísticamente significativa en la incidencia de náusea (RR 0,52, 95\% IC 0,29-0,93, P = 0,03 ), arcada (RR 0,39, 95\% IC 0,18-0,88, P=0,02) y vómito (RR 0,26, 95\% IC 0,11-0,64, P=0,003) en el grupo de pacientes tratados con fentanilo IT comparado con el grupo tratado con ondansetrón EV (Figura 3).

\section{Discusión}

La fisiopatología de IONV está relacionada con factores centrales, periféricos e inducido por fármacos. A nivel central las náuseas y el vómito se pueden desencadenar por estimulación cortical sobre el centro generador del vómito ubicado en la médula espinal. En el período perioperatorio factores como el miedo, el dolor, la ansiedad, las náuseas condicionadas relacionadas con las señales ambientales y la estimulación del sistema vestibular son estímulos centrales que pueden causar NAV[9].

Los cinco principales receptores y neurotransmisores que median la aparición de NV son: muscarínicos $\left(M_{1}\right)$, dopamina $\left(D_{2}\right)$, histamina $\left(H_{1}\right)$, 5-hidroxitriptamina-3 $\left(5-\mathrm{HT}_{3}\right)$ y la sustancia $\mathrm{P}[10]$. Los mecanismos periféricos se relacionan con estimulación gástrica directa que desencadena la liberación de sustancia $P$ y

\begin{tabular}{|c|c|c|c|c|c|c|c|}
\hline \multirow[t]{2}{*}{ Estudio } & \multicolumn{3}{|c|}{ Ondansetrón (EV) } & \multicolumn{3}{|c|}{ Fentanilo (IT) } & \multirow[t]{2}{*}{ Valor P } \\
\hline & $\mathbf{n}$ & mg & NAV & $\mathbf{n}$ & $\mu \mathrm{g}$ & NAV & \\
\hline Gowda (2014)[6] & 60 & 4 & $\begin{array}{l}26,67 \% \\
20,00 \% \\
18,33 \%\end{array}$ & 60 & 12,5 & $\begin{array}{r}11,67 \% \\
5,00 \% \\
3,33 \%\end{array}$ & $\begin{array}{l}0,037 * \\
0,013 * \\
0,008 *\end{array}$ \\
\hline Jain (2015)[7] & 30 & 4 & $\begin{array}{l}12,30 \% \\
10,00 \% \\
27,00 \%\end{array}$ & 30 & 12,5 & $\begin{array}{r}10,00 \% \\
7,00 \% \\
3,00 \%\end{array}$ & Sin reporte \\
\hline Soreingam (2017)[8] & 70 & 4 & $\begin{array}{c}12,87 \% \\
8,58 \% \\
8,58 \%\end{array}$ & 70 & 20 & $\begin{array}{l}7,15 \% \\
4,28 \% \\
4,28 \%\end{array}$ & $\begin{array}{l}0,26 \\
0,30 \\
0,30\end{array}$ \\
\hline
\end{tabular}

NAV: Náuseas, arcadas y vómito; EV: Endovenoso; IT: Intratecal. 
Fentanilo intratecal vs ondansetrón en náuseas y vómito en cesárea - A. F. Caballero-Lozada et al.

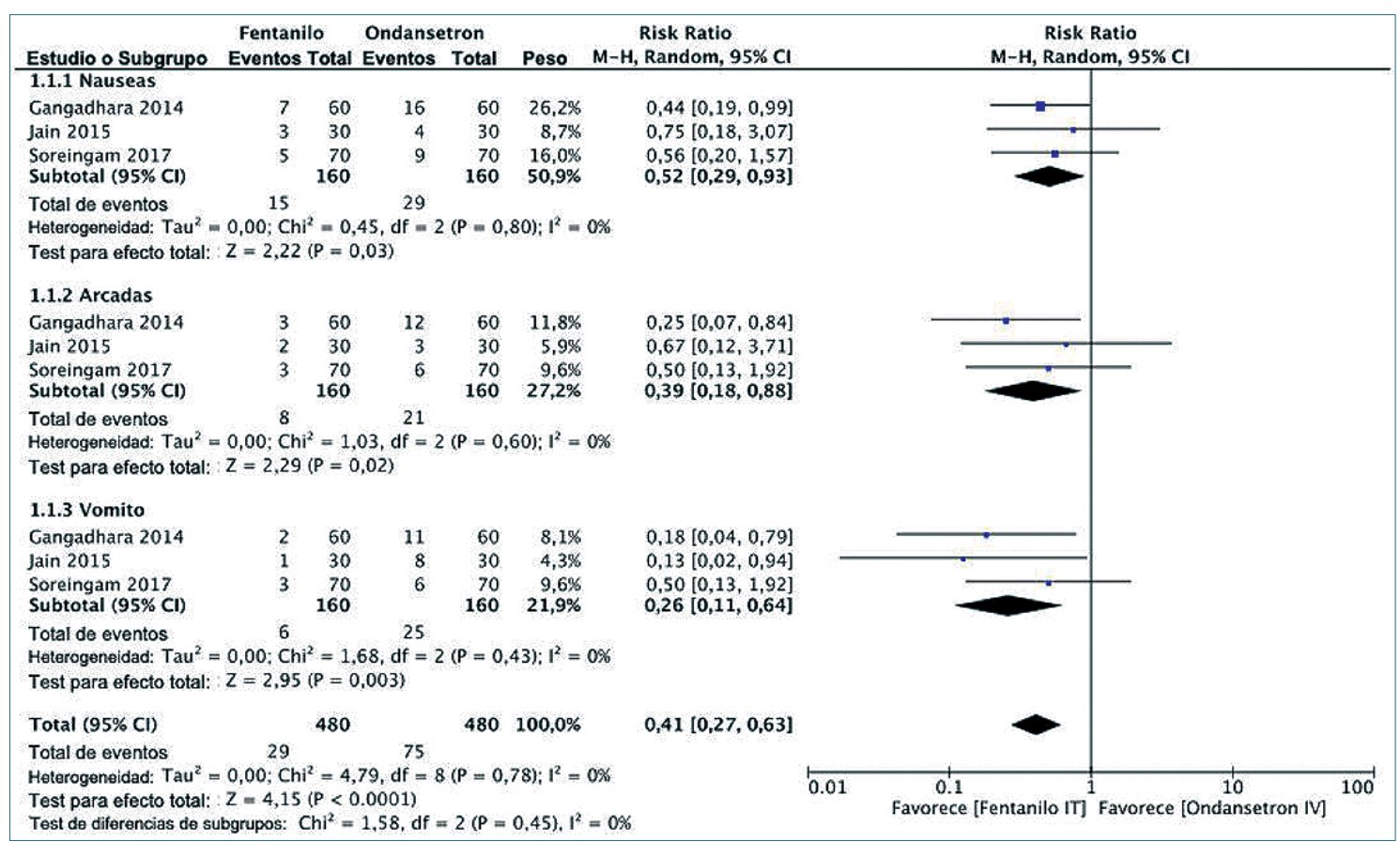

Figura 3. Diagrama de "Forest plot" para las puntuaciones de la incidencia de náuseas, arcadas y vómitos entre los grupos tratados con fentanilo IT vs ondansetrón EV. IT: Intratecal, EV: Endovenoso.

serotonina por las células enterocromafines, estos estimulan las fibras nerviosas vagales y esplácnicas. Las aferencias vagales y esplácnicas terminan en el núcleo del tracto solitario (NTS) y el área postrema (también llamada zona de activación quimiorreceptora) en el tallo cerebral, que son centros relacionados con las NAV, a pesar de que los mecanismos por los cuales los estímulos serotoninérgicos causan NAV no se comprenden completamente[11]. Por otra parte, algunas moléculas como toxinas o fármacos (dentro de ellos los anestésicos y opioides) producen estimulación directa sobre el área postrema a nivel del piso del cuarto ventrículo, desencadenando NAV[12].

Los opioides hidrofílicos como la morfina permanecen durante más tiempo en el líquido cefalorraquídeo (LCR) y pueden ascender rostralmente hasta la zona de activación quimiorreceptora[13]. Por otra parte, los opioides lipofílicos como el fentanilo se absorben rápidamente por la médula espinal. Sin embargo, cuando se utiliza por vía epidural, hasta un $10 \%$ del fentanilo puede ser recuperado por el LCR y alcanzar un nivel cervical[14]. Así mismo la incidencia de NAV intraoperatorias varía según el tipo de opioide y la dosis administrada, siendo mayor con el uso de morfina IT en comparación al fentanilo IT [1]. Aunque algunos estudios encontraron que el uso de fentanilo IT no provocó náuseas[15]

Jelting et al.[1], expone las diferentes posibles causas de NAV intraoperatorias durante cesárea electiva, entre las cuales se encuentra la baja presión arterial que puede causar una perfusión reducida del cerebro, activando el centro de vómitos en el bulbo raquídeo. Además, la paciente gestante tiene una mayor predisposición para el desarrollo de NV debido a los cambios fisiológicos asociados al embarazo y a la manipulación quirúrgica del útero y de las estructuras digestivas contiguas, que pueden promover la liberación de neurotransmisores involucrados en su fisiopatología[10-16]. Por otro lado, en ocasiones es necesario complementar la anestesia regional con una inyección de opiodes sistémicos para obtener un alivio satisfactorio del dolor en la madre. Sin embargo, se ha determinado que dichos opioides sistémicos pueden causar náuseas y vómitos[17]. Los opiáceos lipófilos intratecales como el fentanilo, por su parte, aumentan tanto la duración como la intensidad de la anestesia espinal y disminuyen las NAV intraoperatorias[18], además, de ser un fármaco de bajo costo[19].

Existen varias estrategias farmacológicas para disminuir las IONV, siendo el uso de inhibidores del 
receptor $5-\mathrm{HT}_{3}$ como el ondansetrón una de ellas[1]. El efecto fundamental de la unión de opioides a su receptor en la médula espinal es reducir la liberación de transmisores excitatorios con la consiguiente reducción de la transmisión nociceptiva[20]. El ondansetrón es un antagonista del receptor de serotonina y periféricamente reduce la actividad del nervio vagal, reduciendo las NAV intraoperatorias, con una baja incidencia de efectos secundarios, pero un alto costo de adquisición[21]. Para mejorar la calidad analgésica, se ha implementado el uso de opioides por vía IT, obteniendo un alivio satisfactorio del dolor y disminuyendo los efectos secundarios de la anestesia intraoperatoria[6]-[8]. Se ha visto que la combinación de bupivacaína con fentanilo IT durante la cesárea proporciona una mejor calidad de la anestesia y se asocia con una menor incidencia de efectos secundarios[1],[8]. Lo anterior coincide con los resultados del presente metaanálisis donde se observó una disminución significativa en las NAV intraoperatorias en pacientes que recibieron fentanilo IT, en comparación con pacientes a los que se les administró ondansetrón EV durante cesárea electiva.

Kaya y cols., en el 2007, evaluaron el efecto combinado de la dosis baja de bupivacaína espinal con o sin precarga de coloides y medicamentos opioides como fentanilo, donde observaron una reducción de los efectos secundarios de la anestesia de $70 \%$, encontrando que la dosis baja reduce la hipotensión, náuseas y vómito[22],[23]. Se ha descrito que las dosis bajas de fentanilo $(12,5 \mu \mathrm{g})$ son altamente lipófilas y no permanecen lo suficientemente libres en el líquido cefalorraquídeo para alcanzar la zona gatillo quimiorreceptora (CTZ), en una concentración suficiente para causar náuseas, pero esta misma dosis es suficiente para disminuir la estimulación nociceptiva durante las maniobras de tracción peritoneal y exteriorización del útero lo que lleva a una disminución de náuseas y vómito[7]. En el estudio de Gowda y Dhorigol[6], se encuentra una reducción en la incidencia de náuseas, arcadas y vómitos en el grupo de fentanilo IT con una dosis de $12,5 \mu \mathrm{g}$ en comparación con el grupo de ondansetrón EV y esta diferencia es estadísticamente significativa, con una disminución alrededor de $15 \%$ en las NAV intraoperatorias. Estos hallazgos son similares al estudio de Jain y cols., donde usaron la misma dosis de fentanilo IT y describen menores efectos secundarios, con hasta $25 \%$ menos vómito y $30 \%$ más mujeres que no presentaron náuseas, en comparación con el grupo de ondansetrón EV[7]. Los autores concluyeron que el fentanilo IT es la mejor modalidad para la prevención de náuseas y vómitos en este grupo de pacientes.

En el estudio de Soreingam y cols., no se observaron diferencias estadísticamente significativas en la frecuencia cardíaca y la presión arterial entre el grupo fentanilo IT y el grupo ondansetrón EV después de la anestesia espinal[8]. En dicho estudio, la incidencia de NAV intraoperatorias fue mayor en el grupo de ondansetrón $\mathrm{EV}$ en comparación con el grupo de fentanilo IT, sin embargo, la incidencia de NVPO fue mayor en el grupo de fentanilo IT. Estos hallazgos coinciden con los reportados en estudios anteriores, donde las dosis de fentanilo IT entre 10 a $15 \mu \mathrm{g}$, generan una excelente analgesia intraoperatoria y postoperatoria sin efectos secundarios significativos, en contraposición a los pacientes que recibieron $7,5 \mu \mathrm{g}$ o dosis superiores a $20 \mu \mathrm{g}$ de fentanilo[24]-[26]. La administración de fentanilo IT proporciona una analgesia mejorada y, por lo tanto, disminuye la incomodidad de las manipulaciones intraoperatorias que inician episodios eméticos, lo que podría ser la causa de la reducción de la incidencia de NAV.

Entre las limitaciones de este metaanálisis, se tiene que a pesar de una búsqueda exhaustiva de la literatura, sólo cumplieron criterios de inclusión 3 estudios controlados-aleatorizados, dos de los cuales usaron $12,5 \mu \mathrm{g}$ de fentanilo intratecal y uno utilizó 20 $\mu \mathrm{g}$ de fentanilo. Además, dos de los tres estudios tenían un riesgo de sesgo de selección incierto y uno un riesgo alto para el mismo tipo de sesgo, lo cual afecta directamente la calidad de los resultados de metaanálisis aquí presentados. En general, los resultados obtenidos sugieren que la administración de 12,5 a $20 \mu \mathrm{g}$ de fentanilo IT puede disminuir la incidencia de NAV intraoperatorias en pacientes sometidas a cesárea electiva en comparación a una dosis estándar de ondansetrón EV.

\section{Referencias}

1. Jelting $Y$, Klein $C$, Harlander $T$, Eberhart L, Roewer N, Kranke P.
Preventing nausea and vomiting in women undergoing regional anesthesia for cesarean section: challenges and solutions. Local
Reg Anesth. 2017;10:83-90. https://doi.org/10.2147/LRA. S111459

2. Semiz A, Akpak YK, Yılanlıojlu 
NC, Babacan A, Gönen G, Çam Gönen C, Asıliskender M, Karaküçük S. Prediction of intraoperative nausea and vomiting in caesarean delivery under regional anaesthesia. J Int Med Res. 2017;45(1):332-339. https://doi. rg/10.1177/0300060516680547

3. Balki M, Carvalho JCA. Intraoperative nausea and vomiting during cesarean section under regional anesthesia. Int J Obstet Anesth 2005;14(3):23041. https://doi.org/10.1016/j. ijoa.2004.12.004

4. Griffiths JD, Gyte GM, Paranjothy S, Brown HC, Broughton HK, Thomas J. Interventions for preventing nausea and vomiting in women undergoing regional anaesthesia for caesarean section. Cochrane Database Syst Rev. 2012;9(9):CD007579. https:// doi.org/10.1002/14651858. CD007579.pub2

5. Abouleish El, Rashid S, Haque S, Giezentanner A, Joynton $P$, Chuang AZ. Ondansetron versus placebo for the control of nausea and vomiting during Caesarean section under spinal anaesthesia. Anaesthesia. 1999;54(5):479-482. https:// doi.org/10.1046/j.13652044.1999.00798.x

6. Gangadhara Gowda, Dhorigol M. Comparision of Intrathecal Fentanyl and Intravenous Ondansetron for Prevention of Nausea-Vomiting during Cesarean Delivery with Spinal Anaesthesia. Indian J Public Heal Res Dev 2014;5(4):157-60. https://doi.org/10.5958/09765506.2014.00032.1

7. Jain P, Jigar J, Raval B. Comparision of intrathecal fentanyl and intravenous ondansetron for prevention of perioperative nausea and vomiting during cesarean delivery under spinal anesthesia. Int J Curr Pharm Clin Res 2015;5(2):109-13.
8. Soreingam $K$, Singh $T H$, Thokchom RS, Singh YAK, Meitei AJ, Singh SS. Comparative study of intravenous ondansetron and intrathecal fentanyl for prevention of perioperative nausea and vomiting during caesarean delivery under spinal anaesthesia with hyperbaric bupivacaine. J Dent Med Sci 2017;16(4):22-6. https://doi.org/10.9790/08531604022226

9. Becker DE. Nausea, vomiting, and hiccups: a review of mechanisms and treatment. Anesth Prog 2010;57(4):150-6. https:// doi.org/10.2344/0003-300657.4.150

10. Bashashati M, McCallum RW. Neurochemical mechanisms and pharmacologic strategies in managing nausea and vomiting related to cyclic vomiting syndrome and other gastrointestinal disorders. Eur J Pharmacol 2014;722:7994. https://doi.org/10.1016/j. ejphar.2013.09.075

11. Spiller R. Recent advances in understanding the role of serotonin in gastrointestinal motility in functional bowel disorders: alterations in 5-HT signalling and metabolism in human disease. Neurogastroenterol Motil 2007;19(s2):25-31. https://doi.org/10.1111/j.13652982.2007.00965.x

12. Mitchelson F. Pharmacological Agents Affecting Emesis. Drugs 1992;43(3):295-315. https:// doi.org/10.2165/00003495199243030-00002

13. Borgeat A, Ekatodramis $G$, Schenker CA. Postoperative Nausea and Vomiting in Regional Anesthesia A Review. Anesthesiol J Am Soc Anesthesiol 2003;98(2):530-47. https:// doi.org/10.1097/00000542200302000-00036

14. Gourlay GK, Murphy TM, Plummer JL, Kowalski SR, Cherry DA,
Cousins MJ. Pharmacokinetics of fentanyl in lumbar and cervical CSF following lumbar epidural and intravenous administration. Pain 1989;38(3):253-9. https://doi.org/10.1016/03043959(89)90210-8

15. Liu S, Chiu AA, Carpenter RL, y cols. Fentanyl prolongs lidocaine spinal anesthesia without prolonging recovery. Anesth Analg 1995;80(4):730-4. https:// doi.org/10.1213/00000539199504000-00014

16. Ishiyama T, Yamaguchi T, Kashimoto S, Kumazawa T. Effects of epidural fentanyl and intravenous flurbiprofen for visceral pain during cesarean section under spinal anesthesia. J Anesth. 2001;15(2):69-73. https://doi. org/10.1007/s005400170029

17. Apfel CC, Laara E, Koivuranta M, Greim CA, Roewer N. A simplified risk score for predicting postoperative nausea and vomiting: conclusions from cross-validations between two centers. Anesthesiology. 1999;91(3):693-700. https:// doi.org/10.1097/00000542199909000-00022

18. Chooi C, Cox JJ, Lumb RS, y cols. Techniques for preventing hypotension during spinal anaesthesia for caesarean section. Cochrane Database Syst Rev 2017;8:CD002251. https:// doi.org/10.1002/14651858. CD002251.pub3

19. Manullang TR, Viscomi CM, Pace NL. Intrathecal fentanyl is superior to intravenous ondansetron for the prevention of perioperative nausea during cesarean delivery with spinal anesthesia. Anesth Analg 2000;90(5):1162-6. https:// doi.org/10.1097/00000539200005000-00030

20. Hindle A. Intrathecal opioids in the management of acute postoperative pain. Contin 
Educ Anaesth Crit Care Pain 2008;8(3):81-5. https://doi. org/10.1093/bjaceaccp/mkn016

21. Khalifa OS. A comparative study of prophylactic intravenous granisetron, ondansetron, and ephedrine in attenuating hypotension and its effect on motor and sensory block in elective cesarean section under spinal anesthesia. Ain-Shams J Anaesthesiol 2015;8(2):166-72. https://doi. org/10.4103/1687-7934.156667

22. Kaya $S$, Karaman $H$, Erdogan $\mathrm{H}$, Akyilmaz A, Turhanoglu S. Combined Use of Low-dose Bupivacaine, Colloid Preload and Wrapping of the Legs for
Preventing Hypotension in Spinal Anaesthesia for Caesarean Section. J Int Med Res 2007;35(5):615-25. https:// doi.1177/147323000703500506

23. Roofthooft E, Van de Velde M. Low-dose spinal anaesthesia for Caesarean section to prevent spinal-induced hypotension. Curr Opin Anaesthesiol 2008;21(3):25962. https://doi.org/10.1097/ ACO.0b013e3282ff5e41

24. Ali MA, Ismail S, Sohaib M, Aman A. A double-blind randomized control trial to compare the effect of varying doses of intrathecal fentanyl on clinical efficacy and side effects in parturients undergoing cesarean section. J Anaesthesiol Clin Pharmacol 2018;34(2):221-6.

25. Dahlgren $G$, Hultstrand $C$, Jakobsson J, Norman M, Eriksson EW, Martin H. Intrathecal sufentanil, fentanyl, or placebo added to bupivacaine for cesarean section. Anesth Analg 1997;85(6):1288-93. https:// doi.org/10.1213/00000539199712000-00020

26. Chu CC, Shu SS, Lin SM, y cols. The effect of intrathecal bupivacaine with combined fentanyl in cesarean section. Acta Anaesthesiol Sin 1995;33(3):149-54. 